\title{
The universality theorem for Hecke $L$-functions
}

\author{
by \\ Hidehiko Mishou (Nagoya)
}

1. Introduction. Let $s \in \mathbb{C}$ be a complex number and $\zeta(s)$ be the Riemann zeta-function. In 1975 S. M. Voronin [12] proved the following remarkable result, which is now called the universality theorem for $\zeta(s)$.

VORONIN's THEOREM. Let $0<r<1 / 4$ and $f(s)$ be a continuous function on the disk $|s| \leq r$ such that $f(s) \neq 0$ on $|s| \leq r$ and $f(s)$ is holomorphic in $|s|<r$. Then for every $\varepsilon>0$, we have

$$
\liminf _{T \rightarrow \infty} \frac{m\left(\left\{\tau \in[0, T]\left|\max _{|s| \leq r}\right| \zeta(s+3 / 4+i \tau)-f(s) \mid<\varepsilon\right\}\right)}{T}>0
$$

where $m$ is the Lebesgue measure on $\mathbb{R}$.

Roughly speaking, this theorem asserts that any analytic function can be uniformly approximated by vertical translation of $\zeta(s)$ and the set of all real numbers which give such approximation has a positive lower density.

After Voronin's work, many mathematicians studied the universality property of other zeta-functions. In particular, concerning number fields, A. Reich [11] proved the property for Dedekind zeta-functions and the author [7] proved it for $L$-functions associated with ideal class characters. The aim of this paper is to prove the universality theorem for Hecke $L$-functions, which are defined more generally.

Let $K$ be a finite extension of the rational number field $\mathbb{Q}$ and $\mathfrak{f}$ be an ideal of $K$. Hecke [2] introduced the notion of Grössencharacters $\chi$ modulo $\widetilde{\mathfrak{f}}$ (we state the definition in Section 2). For $\Re s>1$ the Hecke $L$-function with Grössencharacter $\chi$ is defined by the series

$$
L(s, \chi)=\sum_{\mathfrak{a}} \frac{\chi(\mathfrak{a})}{(N(\mathfrak{a}))^{s}}
$$

where $\mathfrak{a}$ runs through all integral ideals in $K$ except 0 and $N(\mathfrak{a})$ is the norm of the ideal $\mathfrak{a}$.

2000 Mathematics Subject Classification: Primary 11M41. 
Theorem. Let $K$ be a finite extension of $\mathbb{Q}, n=[K: \mathbb{Q}]$ and $\chi$ be a Grössencharacter modulo $\widetilde{\mathfrak{f}}$. Set

$$
\sigma_{K}= \begin{cases}1 / 2 & \text { if } K=\mathbb{Q}, \\ 1-1 / n & \text { otherwise. }\end{cases}
$$

Let $C$ be a compact subset in the strip $\sigma_{K}<\Re s<1$ with connected complement and $f(s)$ be a continuous function on $C$ such that $f(s) \neq 0$ on $C$ and $f(s)$ is holomorphic in the interior of $C$. Then for every $\varepsilon>0$,

$$
\liminf _{T \rightarrow \infty} \frac{1}{T} m\left(\left\{\tau \in[0, T]\left|\max _{s \in C}\right| L(s+i \tau, \chi)-f(s) \mid<\varepsilon\right\}\right)>0 .
$$

It is known that there are two methods for the proof of universality: one is Voronin's original proof, which is presented in [3] in detail, and the other is a probabilistic proof due to Bagchi, detailed in [4]. They are quite different, but conditions and lemmas which are necessary are almost the same. In this paper we employ Bagchi's method. The proof is divided into two parts. One is the limit theorem (Proposition 1), and the other is the denseness lemma (Proposition 2). We can easily prove the limit theorem by applying the general limit theorem due to Laurinčikas [5].

On the other hand, to prove the denseness lemma, we need to consider the character sum

$$
\alpha_{p}=\sum_{\mathfrak{p}} \chi(\mathfrak{p})
$$

where $\mathfrak{p}$ runs through prime ideals with degree 1 which divide the prime number $p$. It is essential that there exist infinitely many primes $p$ such that $\left|\alpha_{p}\right| \geq C$ for some positive constant $C>0$ (actually the set of such primes has a positive lower density). We can prove this by using the prime number theorem in algebraic number fields due to T. Mitsui [9]. In the case that $\chi$ are ideal class characters, by applying the class field theory, we can prove it more easily. See [7] for details.

The reason why there is the restriction $\Re s>\sigma_{K}$ in our theorem is that we can prove the mean square estimate

$$
\int_{0}^{T}|L(\sigma+i t, \chi)|^{2} d t=O(T)
$$

only for $\sigma>\sigma_{K}$. This estimate is necessary to prove the limit theorem.

The organization of this paper is as follows. In Section 2 we give the definition of Hecke characters and basic facts on Hecke $L$-functions. We state Proposition 1 (limit theorem) in Section 3. In Section 4 we prove our theorem under the assumption that Proposition 2 holds. The deduction of our theorem from Proposition 2 is essentially the same as Bagchi's argument. In Section 5, we show that Proposition 2 follows from Proposition 5, and we prove Proposition 5 in Section 6. 
The author would like to express his sincere gratitude to Professor Kohji Matsumoto and Professor Yoshio Tanigawa for their encouragement. He would like to express his sincere thanks to Professor Ryutaro Okazaki who kindly indicated that in the proof of Proposition 5 the general case can be reduced to the case that $K / \mathbb{Q}$ is Galois.

2. Background for $L$-functions. In this section we state the definition of Grössencharacters and basic results on Hecke $L$-functions. See Mitsui [8] or [9] for details. Before we define Grössencharacters, we describe some notations and basic facts about number fields for the convenience of the reader.

Let $K$ be a finite extension of $\mathbb{Q}$ of degree $n$. There are exactly $n$ isomorphisms of $K$ into $\mathbb{C}$ :

$$
K \ni \alpha \mapsto \alpha^{(i)} \in K^{(i)}, \quad i=1, \ldots, n .
$$

Among these there are $r_{1}$ real embeddings, denoted by $K^{(1)}, \ldots, K^{\left(r_{1}\right)}$, and $2 r_{2}$ complex embeddings which are pairwise complex conjugate, which we denote by

$$
K^{\left(r_{1}+1\right)}, \ldots, K^{\left(r_{1}+r_{2}\right)}, K^{\left(r_{1}+r_{2}+1\right)}=\overline{K^{\left(r_{1}+1\right)}}, \ldots, K^{(n)}=\overline{K^{\left(r_{1}+r_{2}\right)}} .
$$

For an integral ideal $\mathfrak{f}$ in $K$, define the unit group modulo $\tilde{\mathfrak{f}}$ by

$$
U(\widetilde{\mathfrak{f}})=\{\varepsilon: \text { units of } K \mid \varepsilon \equiv 1(\bmod \tilde{\mathfrak{f}})\}
$$

where $\varepsilon \equiv 1(\bmod \tilde{\mathfrak{f}})$ means both $\varepsilon \equiv 1(\bmod \mathfrak{f})$ and that $\varepsilon$ is totally positive. Dirichlet's unit theorem asserts that there exist $r=r_{1}+r_{2}-1$ units $\eta_{1}, \ldots, \eta_{r}$ and a root of unity $\varrho$ in $K$ such that any $\varepsilon \in U(\widetilde{\mathfrak{f}})$ can be written uniquely as the product

$$
\varepsilon=\varrho^{a} \eta_{1}^{n_{1}} \ldots \eta_{r}^{n_{r}} \quad\left(a, n_{i} \in \mathbb{Z}\right) .
$$

These $\eta_{1}, \ldots, \eta_{r}$ are called fundamental units of $U(\widetilde{\mathfrak{f}})$. They are not uniquely determined, but the absolute value of the determinant

$$
\operatorname{det}\left(e_{i} \log \left|\eta_{j}^{(i)}\right|\right)
$$

does not depend on the choice of $\eta_{j}$, where

$$
e_{i}= \begin{cases}1 & \left(1 \leq i \leq r_{1}\right) \\ 2 & \left(r_{1}+1 \leq i \leq r_{1}+r_{2}\right)\end{cases}
$$

We call the absolute value of this determinant the regulator of $U(\widetilde{\mathfrak{f}})$ and denote it by $R(\widetilde{\mathfrak{f}})$.

For an integral ideal $\mathfrak{f}$ in $K$, we define the sets

$$
\begin{aligned}
I_{\mathfrak{f}} & =\{\mathfrak{a}: \text { ideals of } K \mid(\mathfrak{a}, \mathfrak{f})=1\}, \\
P_{\mathfrak{f}} & =\{(\alpha): \text { principal ideals } \mid \alpha \in K, \alpha \equiv 1(\bmod \widetilde{\mathfrak{f}})\} .
\end{aligned}
$$


These are Abelian groups under ideal multiplication. In particular the factor group $I_{\mathfrak{f}} / P_{\mathfrak{f}}$ is finite. We call it the ideal class group modulo $\widetilde{\mathfrak{f}}$. We denote the order $\left[I_{\mathfrak{f}}, P_{\mathfrak{f}}\right]$ by $h(\widetilde{\mathfrak{f}})$.

Now we define the Hecke character. We take numbers $v_{q}, a_{p}$ subject to the following restrictions:

$$
\begin{cases}a_{p}=0,1, & 1 \leq p \leq r_{1} \\ a_{p} \in \mathbb{Z}, & r_{1}+1 \leq p \leq r_{1}+r_{2}, \\ v_{q} \in \mathbb{R}, & 1 \leq q \leq r_{1}+r_{2} \quad \text { such that } \quad \sum_{q} v_{q}=0 .\end{cases}
$$

Let $K^{*}=K-\{0\}$ and $S^{1}=\{s \in \mathbb{C}|| s \mid=1\}$. Define $\chi_{\infty}: K^{*} \rightarrow S^{1}$ by

$$
\chi_{\infty}(\alpha)=\prod_{q=1}^{r_{1}+r_{2}}\left|\alpha^{(q)}\right|^{i v_{q}} \prod_{p=1}^{r_{1}+r_{2}}\left(\frac{\alpha^{(p)}}{\left|\alpha^{(p)}\right|}\right)^{a_{p}} .
$$

Since $\sum v_{q}=0, \chi_{\infty}$ is trivial on $\mathbb{Q}^{*}=\mathbb{Q}-\{0\}$. We suppose that the kernel of $\chi_{\infty}$ contains the unit group modulo $\tilde{\mathfrak{f}}$, i.e.

$$
\chi_{\infty}(\varepsilon)=1 \quad \text { for } \varepsilon \in U(\widetilde{\mathfrak{f}}) .
$$

Then we can identify $\chi_{\infty}$ with a character on $P_{\mathfrak{f}}$.

If the homomorphism $\chi: I_{\mathfrak{f}} \rightarrow S^{1}$ is identified with $\chi_{\infty}$ on $P_{\mathfrak{f}}$, that is,

$$
\chi(\mathfrak{a})=\chi_{\infty}(\alpha), \quad \mathfrak{a}=(\alpha) \in P_{\mathfrak{f}}, \quad \alpha \equiv 1(\bmod \widetilde{\mathfrak{f}}),
$$

then we call $\chi$ a Grössencharacter modulo $\tilde{\mathfrak{f}}$.

In order to satisfy the condition (3), because of the unit theorem, it is enough to satisfy

$$
\chi_{\infty}\left(\eta_{j}\right)=1
$$

for $j=1, \ldots, r$, and $\chi_{\infty}(\varrho)=1$. This is equivalent to

$$
\sum_{q=1}^{r_{1}+r_{2}} v_{q} \log \left|\eta_{j}^{(q)}\right|+\sum_{p=r_{1}+1}^{r_{1}+r_{2}} a_{p} \arg \eta_{j}^{(p)}=2 \pi m_{j} \quad(j=1, \ldots, r)
$$

for some integers $m_{1}, \ldots, m_{r}$. Adding the condition $\sum v_{q}=0$ we have

$$
\left(\begin{array}{ccc}
1 & \ldots & 1 \\
\log \left|\eta_{1}^{(1)}\right| & \ldots & \log \left|\eta_{1}^{\left(r_{1}+r_{2}\right)}\right| \\
\ldots \ldots \ldots \ldots & \ldots \ldots \ldots \ldots \ldots \ldots \\
\log \left|\eta_{r}^{(1)}\right| & \ldots & \log \left|\eta_{r}^{\left(r_{1}+r_{2}\right)}\right|
\end{array}\right)\left(\begin{array}{c}
v_{1} \\
v_{2} \\
\vdots \\
v_{r}
\end{array}\right)=\left(\begin{array}{c}
0 \\
2 \pi m_{1}-\sum_{p=r_{1}+1}^{r_{1}+r_{2}} a_{p} \Theta_{1}^{(p)} \\
\vdots \\
2 \pi m_{r}-\sum_{p=r_{1}+1}^{r_{1}+r_{2}} a_{p} \Theta_{r}^{(p)}
\end{array}\right)
$$

where $\Theta_{j}^{(p)}=\arg \eta_{j}^{(p)}$. Now we calculate the absolute value of the determinant of the matrix on the left-hand side. Because the norm $N\left(\eta_{j}\right)= \pm 1$ for 
units $\eta_{j}$, we have

$$
\begin{aligned}
& \left|\operatorname{det}\left(\begin{array}{ccc}
1 & \ldots & 1 \\
\log \left|\eta_{1}^{(1)}\right| & \ldots & \log \left|\eta_{1}^{\left(r_{1}+r_{2}\right)}\right| \\
\ldots \ldots \ldots & \ldots \ldots & \ldots \ldots \\
\log \left|\eta_{r}^{(1)}\right| & \ldots & \log \left|\eta_{r}^{\left(r_{1}+r_{2}\right)}\right|
\end{array}\right)\right|
\end{aligned}
$$

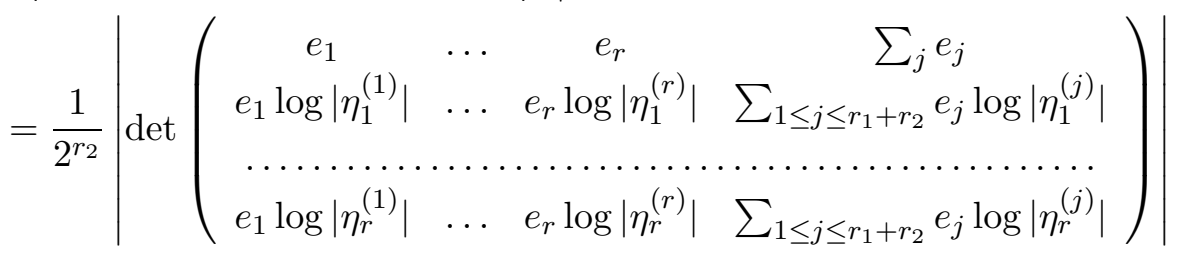

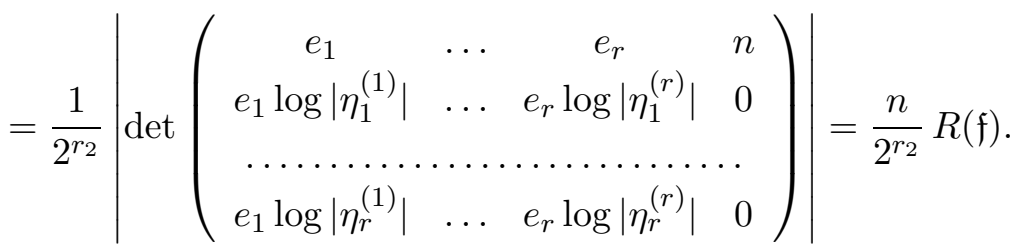

Since the regulator $R(\mathfrak{f}) \neq 0$, the matrix on the left-hand side is regular. Hence if we denote the inverse of this matrix by

$$
\left(\begin{array}{cccc}
\frac{e_{1}}{n} & E_{1}^{(1)} & \ldots & E_{1}^{(r)} \\
\ldots \ldots \ldots \ldots \ldots \ldots \ldots \ldots & \ldots \ldots \\
\frac{e_{r_{1}+r_{2}}}{n} & E_{r_{1}+r_{2}}^{(1)} & \ldots & E_{r_{1}+r_{2}}^{(r)}
\end{array}\right)
$$

then we have the expression

$$
v_{q}=\sum_{j=1}^{r} E_{q}^{(j)}\left(2 \pi m_{j}-\sum_{p=r_{1}+1}^{r_{1}+r_{2}} a_{p} \Theta_{j}^{(p)}\right), \quad q=1, \ldots, r_{1}+r_{2} .
$$

On the other hand, we assume that

$$
\frac{1}{2 \pi} \sum_{p=r_{1}+1}^{r_{1}+r_{2}} a_{p} \arg \varrho^{(p)} \in \mathbb{Z}
$$

for $a_{p}\left(r_{1}+1 \leq p \leq r_{1}+r_{2}\right)$. This is possible because $\chi(\varrho)=1$. For $1 \leq p \leq r_{1}$, we can take the value $a_{p}=0$ or 1 arbitrarily. For $a_{p}$ and $v_{q}$ which satisfy $(7)$ and (8) there exists a Grössencharacter modulo $\widetilde{\mathfrak{f}}$. In particular, if $a_{p}=v_{q}=0, \chi$ is an ideal class group character. If there exists an ideal $\mathfrak{f}_{1} \subset \mathfrak{f}$ and a Grössencharacter $\chi_{1}$ modulo $\tilde{\mathfrak{f}}_{1}$ such that $\chi=\chi_{1}$ on $I_{\mathfrak{f}}$, then we say $\chi$ is induced by $\chi_{1}$. We call $\chi$ primitive, and $\mathfrak{f}$ the conductor of $\chi$, if there does not exist such an $\mathfrak{f}_{1}$.

Next we state basic facts on Hecke $L$-functions. In view of [2], the $L$ function can be analytically continued to the whole $s$-plane, and is holo- 
morphic except at $s=1$. Moreover if $\chi$ is primitive, we have the functional equation. Let

$$
\begin{gathered}
\gamma(\chi)=\prod_{p=r_{1}+1}^{r_{1}+r_{2}} 2^{i v_{p} / 2}, \quad A(\mathfrak{f})=\left(\frac{D N(\mathfrak{f})}{\pi^{n}}\right)^{1 / 2} 2^{-r_{2}}, \\
\Gamma(s, \chi)=\prod_{q=1}^{r_{1}} \Gamma\left(\frac{s+a_{q}-i v_{q}}{2}\right) \prod_{p=r_{1}+1}^{r_{1}+r_{2}} \Gamma\left(s+\frac{\left|a_{p}\right|}{2}-i \frac{v_{q}}{2}\right),
\end{gathered}
$$

where $D=|d|, d$ is the discriminant of $K$, and $\Gamma(s)$ is the gamma function. If $\chi$ is primitive, the function

$$
\xi(s, \chi)=\gamma(\chi) A(\mathfrak{f})^{s} \Gamma(s, \chi) L(s, \chi)
$$

satisfies the functional equation

$$
\xi(1-s, \bar{\chi})=W(\bar{\chi}) \xi(s, \chi)
$$

where $W(\chi)$ is a constant depending on $\chi$ only, with $|W(\chi)|=1$. This fact is due to Hecke. Applying H. S. A. Potter's classical result [10] to this functional equation, we have the estimate

$$
\int_{-T}^{T}|L(\sigma+i t, \chi)|^{2} d t=O(T) \quad\left(\sigma_{K}<\sigma<1\right) .
$$

We note that this estimate also holds for non-primitive $\chi$. Actually, if $\chi_{1}$ is a primitive character which induces $\chi$, then we have

$$
L(s, \chi)=\prod_{\mathfrak{p} \mid \mathfrak{f}}\left(1-\frac{\chi_{1}(\mathfrak{p})}{N(\mathfrak{p})}\right) L\left(s, \chi_{1}\right) .
$$

The finite product on the right-hand side can be estimated by a constant in this region. Hence we have the estimate (9) for any $\chi$.

For $\Re s>1$ we have the Euler product expression

$$
L(s, \chi)=\prod_{\mathfrak{p}}\left(1-\frac{\chi(\mathfrak{p})}{N(\mathfrak{p})}\right)^{-1} .
$$

Infinite product expressions over prime numbers play an important role in the proof of universality. We rewrite the above formula as such an expression. Let

$$
p=\mathfrak{p}_{1}^{x_{1}} \ldots \mathfrak{p}_{z_{p}}^{x_{z_{p}}}, \quad N\left(\mathfrak{p}_{i}\right)=p^{y_{i}},
$$

be the decomposition of the prime $p$ in $K$. Then we have

$$
L(s, \chi)=\prod_{p}\left(\prod_{i=1}^{z_{p}}\left(1-\frac{\chi\left(\mathfrak{p}_{i}\right)}{p^{y_{i} s}}\right)^{-1}\right)=\prod_{p} f_{p}\left(\frac{1}{p^{s}}\right),
$$


say. We note that the functions $f_{p}(z)$ are rational functions of $z$ which have no poles in $|z|<1$. If we expand $f_{p}(z)=1+\sum_{m=1}^{\infty} a_{m}^{(p)} z^{m}$, we have

$$
\left|a_{m}^{(p)}\right| \ll_{\varepsilon} p^{m \varepsilon} \quad \text { for any prime } p \text {. }
$$

3. A limit theorem for $L(s, \chi)$. Let $D=\left\{s \in \mathbb{C} \mid \sigma_{K}<\Re s<1\right\}$. We denote by $H(D)$ the space of analytic functions on $D$ equipped with the topology of uniform convergence on compacta. Let $\mathcal{B}(\mathcal{S})$ stand for the family of Borel subsets of the space $\mathcal{S}$. For $T>0$, define on $(H(D), \mathcal{B}(H(D)))$ the probability measure

$$
P_{T}(A)=\frac{1}{T} m(\{\tau \in[0, T] \mid L(s+i \tau, \chi) \in A\}), \quad A \in \mathcal{B}(H(D)) .
$$

For our purpose, we need a limit theorem which asserts that $P_{T}$ converges weakly to an explicit probability measure as $T$ tends to infinity.

Let $\gamma=\{s \in \mathbb{C}|| s \mid=1\}$ and

$$
\Omega=\prod_{p} \gamma_{p}
$$

where $\gamma_{p}=\gamma$ for all primes $p$. With the product topology and pointwise multiplication, $\Omega$ is a compact Abelian group. So $\Omega$ has a unique probability Haar measure on $(\Omega, \mathcal{B}(\Omega))$. We denote it by $m_{H}$. Let $\omega(p)$ be the projection of $\omega \in \Omega$ to the coordinate space $\gamma_{p}$. We set

$$
L(s, \chi, \omega)=\prod_{p} \prod_{i=1}^{z_{p}}\left(1-\frac{\omega(p)^{y_{i}} \chi\left(\mathfrak{p}_{i}\right)}{p^{y_{i} s}}\right)^{-1} .
$$

We can prove that for almost all $\omega \in \Omega$ this product converges in $H(D)$ the same way as in the proof of [4, Lemma 5.1.6]. Hence this product can be regarded as an $H(D)$-valued random element on $\Omega$. We denote by $P_{L}$ the distribution of $L(s, \omega)$, i.e.

$$
P_{L}(A)=m_{H}(\{\omega \in \Omega \mid L(s, \chi, \omega) \in A\}), \quad A \in \mathcal{B}(H(D)) .
$$

Now we apply Laurinčikas's general limit theorem, which is proved in [6] (see also [5]). Since the assumptions of Laurinčikas's theorem are satisfied by (9), (11) and (12), the following limit theorem for $L(s, \chi)$ holds:

Proposition 1. As $T$ tends to infinity, $P_{T}$ converges weakly to $P_{L}$, i.e.

$$
P_{T} \Rightarrow P_{L} \quad(T \rightarrow \infty) .
$$

By the theory of probability measures, this proposition implies that for any open subset $G$ in $H(D)$,

$$
\liminf _{T \rightarrow \infty} P_{T}(G) \geq P_{L}(G) .
$$


4. Outline of the proof of the Theorem. In view of the statement of the limit theorem, we need to consider the product

$$
L(s, \chi, \omega)=\prod_{p} \prod_{i=1}^{z_{p}}\left(1-\frac{\chi\left(\mathfrak{p}_{i}\right) \omega_{p}}{p^{y_{i} s}}\right)^{-1} \quad(\omega \in \Omega) .
$$

For $a_{p} \in \gamma$ we set

$$
f_{p}\left(s, a_{p}\right)=-\sum_{i=1}^{z_{p}} \log \left(1-\frac{a_{p}^{y_{i}} \chi\left(\mathfrak{p}_{i}\right)}{p^{y_{i} s}}\right) .
$$

Then we have

$$
\log L(s, \chi, \omega)=\sum_{p} f_{p}\left(s, \omega_{p}\right) .
$$

Proposition 2. The set of all series

$$
\sum_{p} f_{p}\left(s, a_{p}\right) \quad\left(a_{p} \in \gamma\right)
$$

which are convergent in $H(D)$ is dense in $H(D)$.

Let us suppose that this proposition holds. Let $S_{L}$ be the support of the probability measure $P_{L}$, that is, the closure of the set

$$
\left\{g(s) \in H(D) \mid P_{L}(G)>0(G \text { : open neighbourhood of } g)\right\} .
$$

Then we have the following lemma.

Lemma 1.

$$
S_{L}=\{g(s) \in H(D) \mid g(s) \neq 0(s \in D)\} \cup\{0\} .
$$

The proof can be obtained from (15), Proposition 1 and Hurwitz's lemma ([4, Theorem 3.4.5]). For the details, refer to the proof of [4, Lemma 6.5.5].

Now we prove the Theorem. Let $f(s)$ satisfy the assumption of the Theorem. First we suppose that $f(s)$ is analytically continued to $D$ and has no zero in $D$. Then by Lemma $1, f(s) \in S_{L}$. Hence for the set

$$
G=\left\{g(s) \in H(D)\left|\max _{s \in C}\right| g(s)-f(s) \mid<\varepsilon\right\}
$$

we have $P_{L}(G)>0$. On the other hand, by (13) we have

$$
\liminf _{T \rightarrow \infty} P_{T}(G) \geq P_{L}(G)
$$

therefore

$$
\liminf _{T \rightarrow \infty} \frac{1}{T} m\left(\left\{\tau \in[0, T]\left|\max _{s \in C}\right| L(s+i \tau, \chi)-f(s) \mid<\varepsilon\right\}\right)>0 .
$$

Next we consider the general case. We quote the following classical result.

Proposition 3 (Mergelyan, 1951). Let $C$ be a compact subset of $\mathbb{C}$ with connected complement, and let $f(s)$ be a continuous function on $C$ which is 
analytic in the interior of $C$. Then for any $\varepsilon>0$ there exists a polynomial $p(s)$ such that

$$
\max _{s \in C}|f(s)-p(s)|<\varepsilon .
$$

The proof is given in [13]. Since $f(s) \neq 0$ on $C, \log f(s)$ can be defined and is continuous on $C$ and analytic in the interior of $C$. Hence by Proposition 3 there exists a polynomial $p(s)$ such that

$$
\max _{s \in C}|\log f(s)-p(s)|<\varepsilon \text {. }
$$

Then we have

$$
\max _{s \in C}\left|f(s)-e^{p(s)}\right| \leq \max _{s \in C}|f(s)| \max _{s \in C}\left|1-e^{|\log f(s)-p(s)|}\right|<c \varepsilon .
$$

We note that $e^{p(s)}$ has no zero in $D$. Hence by the above argument

$$
\liminf _{T \rightarrow \infty} \frac{1}{T} m\left(\left\{\tau \in[0, T]\left|\max _{s \in C}\right| L(s+i \tau, \chi)-e^{p(s)} \mid<\varepsilon\right\}\right)>0 .
$$

By this formula and (17), we obtain (16). Thus we have deduced the Theorem from Proposition 2.

5. The proof of Proposition 2. The series expansion of $f_{p}\left(s, a_{p}\right)$ in (14) can be expressed as

$$
\begin{aligned}
f_{p}\left(s, a_{p}\right) & =-\sum_{i=1}^{z_{p}} \log \left(1-\frac{\chi\left(\mathfrak{p}_{i}\right) a_{p}^{y_{i}}}{p^{y_{i} s}}\right) \\
& =\sum_{i=1}^{z_{p}} \sum_{k=1}^{\infty} \frac{a_{p}^{k y_{i}} \chi^{k}\left(\mathfrak{p}_{i}\right)}{k p^{k y_{i} s}}=a_{p} \frac{\alpha_{p}}{p^{s}}+g_{p}\left(s, a_{p}\right)
\end{aligned}
$$

where we have set

$$
\alpha_{p}=\sum_{\substack{i=1 \\ y_{i}=1}}^{z_{p}} \chi\left(\mathfrak{p}_{i}\right), \quad g_{p}\left(s, a_{p}\right)=\sum_{y_{i}=1} \sum_{k=2}^{\infty} \frac{a_{p}^{k} \chi^{k}\left(\mathfrak{p}_{i}\right)}{p^{k s}}+\sum_{y_{i}>1} \sum_{k=1}^{\infty} \frac{a_{p}^{k y_{i}} \chi^{k}\left(\mathfrak{p}_{i}\right)}{p^{k y_{i} s}}
$$

First we show that Proposition 2 follows from the next proposition.

Proposition 4. Fix a $p_{0}>0$. Then the set of all series

$$
\sum_{p>p_{0}} a_{p} \frac{\alpha_{p}}{p_{s}} \quad\left(a_{p} \in \gamma\right)
$$

which are convergent in $H(D)$ is dense in $H(D)$.

Let $g \in H(D), C$ be a compact subset of $D$, and $\varepsilon>0$. By the definition of $g_{p}\left(s, a_{p}\right)$, for arbitrary $\left\{a_{p}\right\} \subset \gamma$,

$$
\sum_{p} \max _{s \in C}\left|g_{p}\left(s, a_{p}\right)\right| \ll \sum_{p} \frac{1}{p^{2 \sigma}}<\infty
$$


since $\sigma>1 / 2$ for $s \in D$. Hence there exists a $p_{0}>0$ such that

$$
\sum_{p>p_{0}} \max _{s \in K}\left|g_{p}\left(s, a_{p}\right)\right|<\varepsilon / 2 .
$$

The function $g(s)-\sum_{p \leq p_{0}} f_{p}(s, 1)$ belongs to $H(D)$. Thus by Proposition 4 we can take $\left\{\bar{a}_{p}\right\}_{p>p_{0}}$ such that

$$
\max _{s \in K}\left|g(s)-\sum_{p \leq p_{0}} f_{p}(s, 1)-\sum_{p>p_{0}} \bar{a}_{p} \frac{\alpha_{p}}{p^{s}}\right|<\varepsilon / 2 .
$$

We set

$$
a_{p}= \begin{cases}1 & \left(p \leq p_{0}\right), \\ \bar{a}_{p} & \left(p>p_{0}\right) .\end{cases}
$$

Then in view of $(21)$ and (22) we have

$$
\begin{aligned}
& \max _{s \in K}\left|g(s)-\sum_{p} f_{p}\left(s, a_{p}\right)\right| \\
& \quad=\max _{s \in K}\left|g(s)-\sum_{p \leq p_{0}} f_{p}(s, 1)-\sum_{p>p_{0}} \bar{a}_{p} \frac{\alpha_{p}}{p^{s}}-\sum_{p>p_{0}} g_{p}\left(s, \bar{a}_{p}\right)\right| \\
& \quad \leq \max _{s \in K}\left|g(s)-\sum_{p \leq p_{0}} f_{p}(s, 1)-\sum_{p>p_{0}} \bar{a}_{p} \frac{\alpha_{p}}{p^{s}}\right|+\sum_{p>p_{0}} \max _{s \in K}\left|g_{p}\left(s, a_{p}\right)\right| \\
& <\varepsilon / 2+\varepsilon / 2=\varepsilon .
\end{aligned}
$$

Therefore Proposition 2 follows from Proposition 4. In order to prove Proposition 4, we apply the following general denseness lemma due to Bagchi ([1, Theorem 6.5.10]).

Lemma 2. Let $D$ be a connected domain and $\left\{f_{m}\right\}$ be a sequence in $H(D)$ which satisfies:

(a) If $\mu$ is a complex Borel measure on $(\mathbb{C}, \mathcal{B}(\mathbb{C}))$ with a compact support contained in $D$ such that $\sum_{m=1}^{\infty}\left|\int_{\mathbb{C}} f_{m} d \mu\right|<\infty$, then $\int_{\mathbb{C}} s^{r} d \mu(s)=0$ for any non-negative integer $r$.

(b) The series $\sum_{m} f_{m}$ converges in $H(D)$.

(c) For any compact subset $K \subset D, \sum_{m=1}^{\infty} \sup _{s \in K}\left|f_{m}(s)\right|^{2}<\infty$.

Then the set of all convergent series

$$
\sum_{m=1}^{\infty} a_{m} f_{m} \quad\left(a_{m} \in \gamma\right)
$$

is dense in $H(D)$.

We apply this lemma to $\left\{\alpha_{p} / p^{s}\right\}$. We note that if the conditions hold for $p_{0}=1$ then they also hold for any $p_{0}>1$. Hence it is enough to prove Proposition 4 for $p_{0}=1$. 
As we state in Section $3, L(s, \omega)$ converges for almost all $\omega \in \Omega$. This implies that there exists an element $\left\{\bar{a}_{p}\right\} \in \Omega$ such that

$$
\log L\left(s, \bar{a}_{p}\right)=\sum_{p} f_{p}\left(s, \bar{a}_{p}\right)
$$

converges. Because of this and (20), the series $\sum_{p} \bar{a}_{p} \alpha_{p} / p^{s}$ converges in $H(D)$. It is sufficient to prove Proposition 4 for $\left\{\bar{a}_{p} \alpha_{p} / p^{s}\right\}$ instead of $\left\{\alpha_{p} / p^{s}\right\}$. Hence the condition (b) holds. We note that $\sigma>1 / 2$ for $s \in D$. Thus

$$
\sum_{p}\left(\max _{s \in K}\left|\frac{\alpha_{p}}{p^{s}}\right|\right)^{2} \ll \sum_{p} \frac{1}{p^{2 \sigma}}<\infty,
$$

so the condition (c) holds.

It remains to verify the condition (a). We shall prove that if $\mu$ is a complex Borel measure on $(\mathbb{C}, \mathcal{B}(\mathbb{C}))$ with compact support in $D$ such that

$$
\sum_{p}\left|\int_{\mathbb{C}} \frac{\alpha_{p}}{p^{s}} d \mu(s)\right|<\infty
$$

then

$$
\int_{\mathbb{C}} s^{r} d \mu(s)=0 \quad \text { for } r \geq 0 .
$$

Putting

$$
\varrho(z)=\int_{\mathbb{C}} e^{-s z} d \mu(s) \quad \text { for } z \in \mathbb{C},
$$

we can rewrite (23) as

$$
\sum_{p}\left|\alpha_{p}\right| \varrho(\log p) \mid<\infty
$$

We note

$$
\varrho^{(r)}(0)=\int_{\mathbb{C}} s^{r} d \mu(s) .
$$

Hence to prove (a), it is enough to show that

$$
\varrho(z) \equiv 0
$$

when (25) holds.

To verify (26), we apply two lemmas for entire functions of exponential type. Recall that an entire function $f(s)$ is of exponential type if

$$
\limsup _{T \rightarrow \infty} \frac{\log \left|f\left(r e^{i \theta}\right)\right|}{r}<\infty
$$

uniformly in $\theta,|\theta| \leq \pi$. 
Lemma 3. Let $\mu$ be a complex measure on $(\mathbb{C}, \mathcal{B}(\mathbb{C}))$ with compact support contained in the half-plane $\sigma>\sigma_{0}$. Let

$$
f(s)=\int_{\mathbb{C}} e^{s z} d \mu(z) .
$$

If $f \not \equiv 0$ then

$$
\limsup _{r \rightarrow \infty} \frac{\log |f(r)|}{r}>\sigma_{0} .
$$

Lemma 4. Let $f(s)$ be an entire function of exponential type and $\left\{\lambda_{m}\right\}$ a sequence of complex numbers. Let $\alpha, \beta$ and $\delta$ be positive numbers such that

(a) $\limsup _{y \rightarrow \infty} \frac{\log |f( \pm i y)|}{y} \leq \alpha$,

(b) $\left|\lambda_{m}-\lambda_{n}\right| \geq \delta|m-n|(m, n \in \mathbb{N})$,

(c) $\lim _{m \rightarrow \infty} \frac{\lambda_{m}}{m}=\beta$,

(d) $\alpha \beta<\pi$.

Then

$$
\limsup _{m \rightarrow \infty} \frac{\log \left|f\left(\lambda_{m}\right)\right|}{\left|\lambda_{m}\right|}=\limsup _{r \rightarrow \infty} \frac{\log |f(r)|}{r} .
$$

In the case of the Riemann zeta-function, we have

$$
\sum_{p}|\varrho(\log p)|<\infty
$$

instead of (25). We can prove (26) for the Riemann zeta-function from this inequality by applying the above two lemmas and the classical estimate

$$
\sum_{p<x} \frac{1}{p}=\log \log x+O(\exp (-c \sqrt{\log x}))
$$

But in the present case we need to consider the character sum $\alpha_{p}$ defined by (19). We can deduce the following proposition from the theory of Hecke $L$-functions.

Proposition 5. Assume that $K$ is a finite extension of $\mathbb{Q}$ with degree $n$ and $\chi$ is a Grössencharacter. For any $\varepsilon>0$ there exists a certain condition $(*)$ on primes such that

1. For a prime number $p$ which satisfies $(*)$ we have

$$
\left|\alpha_{p}\right| \geq n-\varepsilon
$$

where $n=[K: \mathbb{Q}]$.

2. The number of primes which satisfy $(*)$ and are less than $x$ equals

$$
c_{0} \int_{2}^{x} \frac{d t}{\log t}+O\left(x \exp \left(-c_{1} \sqrt{\log x}\right)\right),
$$

where $c_{0}$ and $c_{1}$ are positive constants depending on $\varepsilon$ and $\chi$. 
REMARK 1 . In the case that $K$ is totally real Galois, the condition (*) is the condition $(* 6)$ in Section 6 . In the case that $K$ is totally imaginary Galois, it is the condition $(* * 6)$. And in general case it is the condition (!5).

By Abel summation, Proposition 5 gives the formula

$$
\sum_{p}^{*} \frac{1}{p}=c_{0} \log \log x+c_{2}+O\left(\frac{1}{\log ^{2} x}\right)
$$

where $\sum^{*}$ denotes the sum running over primes satisfying the condition $(*)$. Now we show (26) under the assumption that Proposition 5 holds. We take a sufficiently large $N>0$ such that the support of $\mu$ is contained in the region $\{s \in \mathbb{C}|1 / 2<\sigma<1,| \Im s \mid<N\}$. Then for any $y>0$,

$$
|\varrho( \pm i y)| \leq e^{N y}\left|\int_{\mathbb{C}} d \mu(s)\right|=e^{N y} .
$$

We set $\alpha=N$. We fix $\beta>0$ such that $\beta<\pi / N$.

By (25) and Proposition 5 we have

$$
\infty>\sum_{p}\left|\alpha_{p}\right||\varrho(\log p)|>\sum_{p}^{*}\left|\alpha_{p}\right| \varrho(\log p)\left|>\sum_{p}^{*}\right| \varrho(\log p) \mid .
$$

Consider the set

$$
A=\left\{m \in \mathbb{N}|| \varrho(r) \mid \leq e^{-r} \text { for some } r \in\left(\left(m-\frac{1}{4}\right) \beta,\left(m+\frac{1}{4}\right) \beta\right]\right\} .
$$

Then we find that

$$
\infty>\sum_{p}^{*}|\varrho(\log p)| \geq \sum_{m \notin A} \sum_{p, m}^{*}|\varrho(\log p)|>\sum_{m \notin A} \sum_{p, m}^{*} \frac{1}{p}
$$

where $\sum_{p, m}^{*}$ denotes the sum over primes $p$ satisfying $(*)$ and $(m-1 / 4) \beta<$ $\log p \leq(m+1 / 4) \beta$. Using (27) we have

$$
\begin{aligned}
\sum_{p, m}^{*} \frac{1}{p} & =c_{0} \log \left\{\left(m+\frac{1}{4}\right) \beta\right\}-c_{0} \log \left\{\left(m-\frac{1}{4}\right) \beta\right\}+O\left(\frac{1}{m^{2}}\right) \\
& =\frac{c_{0}}{2 m}+O\left(\frac{1}{m^{2}}\right) .
\end{aligned}
$$

From this it follows that

$$
\sum_{m \notin A} \frac{1}{m}<\infty .
$$

Let $A=\left\{a_{m} \mid a_{1}<a_{2}<\ldots\right\}$. Then, taking into account (29), we obtain

$$
\lim _{m \rightarrow \infty} \frac{a_{m}}{m}=1 \text {. }
$$


By the definition of the set $A$, there exists a sequence $\left\{\lambda_{m}\right\}$ such that

$$
\left(a_{m}-\frac{1}{4}\right) \beta<\lambda_{m} \leq\left(a_{m}+\frac{1}{4}\right) \beta
$$

and

$$
\left|\varrho\left(\lambda_{m}\right)\right| \leq e^{-\lambda_{m}}
$$

In view of (30),

$$
\lim _{m \rightarrow \infty} \frac{\lambda_{m}}{m}=\beta
$$

and we can choose a sufficiently small $\delta>0$ such that $\left|\lambda_{m}-\lambda_{n}\right| \geq \delta|m-n|$ for any $m, n$. Since all hypotheses in Lemma 4 are valid for $\left\{\lambda_{m}\right\}, \alpha, \beta$ and $\delta$, by the inequality (31),

$$
\limsup _{r \rightarrow \infty} \frac{\log |\varrho(r)|}{r}=\limsup _{m \rightarrow \infty} \frac{\log \left|\varrho\left(\lambda_{m}\right)\right|}{\left|\lambda_{m}\right|} \leq-1 .
$$

We note that the support of $\mu$ is contained in the half-plane $\sigma>1 / 2$. Hence if $f \not \equiv 0$, by Lemma 3 we have

$$
\limsup _{r \rightarrow \infty} \frac{\log |\varrho(r)|}{|r|}>-1 .
$$

This inequality clearly contradicts (32). Therefore (26) is valid. Thus we have deduced Proposition 2 from Proposition 5.

6. The proof of Proposition 5. First we recall the definition of Grössencharacters. From $(2)$ and $(4)$, for $\chi(\bmod \widetilde{\mathfrak{f}})$ and $\mathfrak{a}=(\alpha), \alpha \equiv 1(\bmod \widetilde{\mathfrak{f}})$,

$$
\chi(\mathfrak{a})=\chi_{\infty}(\alpha)=\prod_{q=1}^{r_{1}+r_{2}}\left|\alpha^{(q)}\right|^{i v_{q}} \prod_{p=1}^{r_{1}+r_{2}}\left(\frac{\alpha^{(p)}}{\left|\alpha^{(p)}\right|}\right)^{a_{p}},
$$

where $a_{p}$ are integers satisfying (8) and

$$
v_{q}=\sum_{j=1}^{r} E_{q}^{(j)}\left(2 \pi m_{j}-\sum_{p=r_{1}+1}^{r_{1}+r_{2}} a_{p} \Theta_{j}^{(p)}\right) \quad\left(q=1, \ldots, r_{1}+r_{2}\right) .
$$

Putting

$$
\begin{gathered}
W_{q}(\alpha)=\sum_{p=1}^{r_{1}+r_{2}} E_{p}^{(q)} \log \left|\alpha^{(p)}\right| \quad(q=1, \ldots, r), \\
\Theta_{p}(\alpha)=\frac{1}{2 \pi}\left\{\arg \alpha^{(p)}-\sum_{j=1}^{r} \Theta_{j}^{(p)} W_{j}(\alpha)\right\} \quad\left(p=r_{1}+1, \ldots, r_{1}+r_{2}\right),
\end{gathered}
$$


we have the expression

$$
\chi(\mathfrak{a})=\exp \left\{2 \pi i \sum_{q=1}^{r} m_{q} W_{q}(\alpha)+2 \pi i \sum_{p=r_{1}+1}^{r_{1}+r_{2}} a_{p} \Theta_{p}(\alpha)\right\} .
$$

We call $\omega \in K$ a prime ideal number if the principal ideal $(\omega)$ is a prime ideal. Concerning (35), Mitsui [8] showed a certain kind of prime number theorem. In particular, if in Mitsui's theorem we take $\widehat{\varrho}=1$ (in his notation), then we have the following proposition, which is essential to our proof.

Proposition 6. Let $x>0$ and let $\mathfrak{f}$ be an integral ideal such that $N(\mathfrak{f}) \leq$ $(\log x)^{A}(A>0)$. Let $\left\{\alpha_{q}\right\},\left\{\alpha_{q}^{\prime}\right\},\left\{\beta_{p}\right\},\left\{\beta_{p}^{\prime}\right\}$ be real sequences such that

$$
\begin{array}{ll}
0<\alpha_{q}-\alpha_{q}^{\prime} \leq 1 & (q=1, \ldots, r), \\
0<\beta_{p}-\beta_{p}^{\prime} \leq 1 & \left(p=r_{1}+1, \ldots, r_{1}+r_{2}\right) .
\end{array}
$$

Fix fundamental units in $U(\widetilde{\mathfrak{f}})$ and consider $W_{q}$ and $\Theta_{p}$ for them. Denote by $\pi\left(x, \alpha_{q}, \alpha_{q}^{\prime}, \beta_{p}, \beta_{p}^{\prime}\right)$ the number of prime ideal numbers $\omega$ in $K$ which satisfy the conditions

$$
\begin{cases}\omega \equiv 1(\bmod \tilde{\mathfrak{f}}), & |N(\omega)| \leq x \\ \alpha_{q}^{\prime} \leq W_{q}(\omega)<\alpha_{q} & (q=1, \ldots, r) \\ \beta_{p}^{\prime} \leq \Theta_{p}(\omega)<\beta_{p} & \left(p=r_{1}+1, \ldots, r_{1}+r_{2}\right)\end{cases}
$$

Then

$$
\begin{aligned}
& \pi\left(x, \alpha_{q}, \alpha_{q}^{\prime}, \beta_{p}, \beta_{p}^{\prime}\right) \\
& \quad=\prod_{q=1}^{r}\left(\alpha_{q}-\alpha_{q}^{\prime}\right) \prod_{p=r_{1}+1}^{r_{1}+r_{2}}\left(\beta_{p}-\beta_{p}^{\prime}\right) \frac{w(\widetilde{\mathfrak{f}})}{h \widetilde{(\mathfrak{f})}} \int_{2}^{x} \frac{d t}{\log t}+O\left(x e^{-c \sqrt{\log x}}\right)
\end{aligned}
$$

where $h(\widetilde{\mathfrak{f}})$ is the class number modulo $\widetilde{\mathfrak{f}}, w(\widetilde{\mathfrak{f}})$ is the number of roots of unity in $U(\widetilde{\mathfrak{f}})$ and the $O$-constant depends only on $A$.

First we suppose that $K$ is Galois. The key point is that the prime ideals $\mathfrak{p}_{i}$ in (10) are conjugate to each other for the action of the Galois group $\operatorname{Gal}(K / \mathbb{Q})$ if $K$ is Galois. In general case, by taking the Galois closure of $K$ instead of $K$, we can easily reduce the problem to the case that $K$ is Galois. The general case will be discussed later.

We further split the argument into two cases: $K$ totally real and $K$ totally imaginary.

First we consider the case of $K$ totally real Galois. Since $r_{1}=n$ and $r_{2}=0$, for $\mathfrak{a}=(\alpha)$ with $\alpha \equiv 1(\bmod \widetilde{\mathfrak{f}})$ we have

$$
\chi(\mathfrak{a})=\prod_{q=1}^{n}\left|\alpha^{(q)}\right|^{i v_{q}}
$$


Suppose that a prime number $p$ completely splits in $K$ :

$$
p=\mathfrak{p}_{1} \ldots \mathfrak{p}_{n}, \quad N\left(\mathfrak{p}_{i}\right)=p,
$$

and $\mathfrak{p}_{1}$ belongs to $P_{\mathfrak{f}}$. Let $\omega \in K$ be such that $\omega \equiv 1(\bmod \widetilde{\mathfrak{f}})$ and $(\omega)=\mathfrak{p}_{1}$. Since $K / \mathbb{Q}$ is Galois, if we set $G=\operatorname{Gal}(K / \mathbb{Q})$, then any $\mathfrak{p}_{i}$ can be expressed as

$$
\mathfrak{p}_{i}=(\sigma(\omega)) \quad \text { for each } \sigma \in G .
$$

Hence for such $p$ we have

$$
\alpha_{p}=\sum_{\sigma \in G} \chi((\sigma(\omega)))
$$

Here we note that $\chi((\omega))=\chi_{\infty}(\omega)$ since $\omega \equiv 1(\bmod \widetilde{\mathfrak{f}})$. However the relation $\sigma(\omega) \equiv 1(\bmod \tilde{\mathfrak{f}})$ does not always hold for any $\sigma \neq 1$. We put $\mathfrak{f}^{\prime}=(N(\mathfrak{f}))$ and assume

$$
\omega \equiv 1\left(\bmod \widetilde{\mathfrak{f}}^{\prime}\right) .
$$

Then $\sigma(\omega) \equiv 1\left(\widetilde{\bmod }_{\tilde{\mathfrak{f}}^{\prime}}\right)$ for any $\sigma \in G$. Since $\mathfrak{f} \mid \mathfrak{f}^{\prime}, \chi$ can be regarded as a character modulo $\widetilde{\mathfrak{f}}^{\prime}$. Hence for any $\sigma \in G$,

$$
\chi((\sigma(\omega)))=\chi_{\infty}(\sigma(\omega)),
$$

and they are expressed in the form (36).

In view of $(1), \omega$ corresponds to $\left(\omega^{(1)}, \ldots, \omega^{(n)}\right)$. Similarly $\sigma(\omega)$ corresponds to $\left(\sigma\left(\omega^{(1)}\right), \ldots, \sigma\left(\omega^{(n)}\right)\right)$. For any $\sigma$ and $i$ there exists a $j$ such that $\sigma\left(\omega^{(i)}\right)=\omega^{(j)}$. We denote this $j$ by $\sigma(i)$. Then $\sigma(\omega)$ corresponds to $\left(\omega^{\sigma(1)}, \ldots, \omega^{\sigma(n)}\right)$ and $\sigma$ can be regarded as an element of $S_{n}$. Thus

$$
\chi_{\infty}(\sigma(\omega))=\prod_{q=1}^{n}\left|\omega^{\sigma(q)}\right|^{i v_{q}}=\prod_{q=1}^{n}\left|\omega^{(q)}\right|^{i v_{\sigma^{-1}(q)}} .
$$

Let $p$ be a prime number which satisfies the condition

$$
(* 1) \quad\left\{\begin{array}{l}
p \text { completely splits in } K \text { as } p=\mathfrak{p}_{1} \ldots \mathfrak{p}_{n} \\
\text { and } \mathfrak{p}_{i} \in P_{\mathfrak{f}} \text { for } 1 \leq i \leq n,
\end{array}\right.
$$

and let $\omega$ be a prime ideal number such that $\mathfrak{p}_{1}=(\omega)$ and $\omega \equiv 1\left(\bmod \widetilde{\mathfrak{f}}^{\prime}\right)$. Then, taking into account (37) and (39), we have

$$
\begin{aligned}
\alpha_{p} & =\sum_{\sigma \in G} \chi_{\infty}(\sigma(\omega))=\sum_{\sigma \in G} \prod_{q=1}^{n}\left|\omega^{(q)}\right|^{i v_{\sigma(q)}} \\
& =\sum_{\sigma \in G} \exp \left\{i \sum_{q=1}^{n} v_{\sigma(q)} \log \left|\omega^{(q)}\right|\right\} .
\end{aligned}
$$


Hence for such $p$,

$$
\left|\alpha_{p}\right|=\left|1+\sum_{\sigma \neq 1} \exp \left(i \sum_{q=1}^{n}\left(v_{\sigma(q)}-v_{q}\right) x_{q}\right)\right|,
$$

where we put $\log \left|\omega^{(q)}\right|=x_{q}$.

Now we find the conditions on $\omega$ under which the right-hand side of (40) is sufficiently close to $n$. For any $\varepsilon>0$ we can choose $\delta>0$ such that if

$$
-\delta \leq \sum_{q=1}^{n}\left(v_{\sigma(q)}-v_{q}\right) x_{q} \leq \delta \quad \text { for } \sigma \neq 1,
$$

then

$$
|| \alpha_{p}|-n|<\varepsilon .
$$

Because of the definition of $v_{q}$ we have

$$
v_{\sigma(n)}=-\left(v_{\sigma(1)}+\ldots+v_{\sigma(n-1)}\right) \quad(\sigma \in G) .
$$

Substituting this relation into (41) we have

$$
-\delta \leq \sum_{q=1}^{n-1}\left(v_{\sigma(q)}-v_{q}\right) y_{q} \leq \delta \quad \text { for } \sigma \neq 1,
$$

where we have put $y_{q}=x_{q}-x_{n}$.

On the other hand, the definition (33) of $W_{q}(\omega)$ is

$$
W_{q}(\omega)=\sum_{p=1}^{n} E_{p}^{(q)} x_{p} \quad(q=1, \ldots, n-1) .
$$

By the definition (6) of $\left(E_{p}^{(q)}\right)$ in Section 2, we have

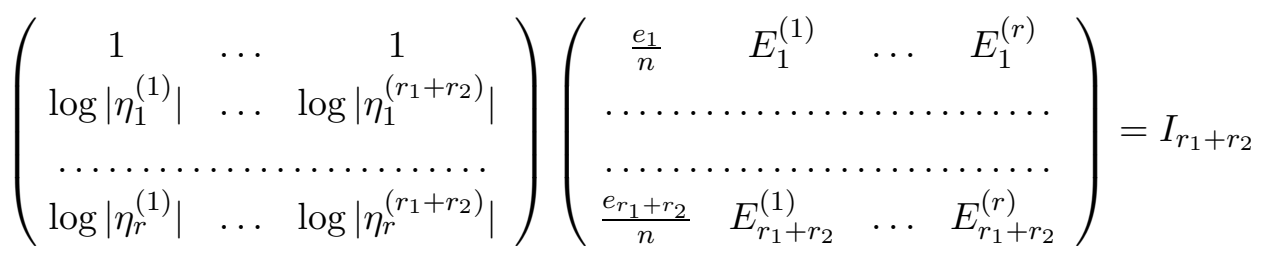

where $I_{r_{1}+r_{2}}$ denotes the unit matrix. It follows that $\sum_{p} E_{p}^{(q)}=0$ for any $q$ and the matrix $\left(E_{p}^{(q)}\right)_{p, q=1}^{r}$ is regular. Substituting these relations into (33) we have

$$
W_{q}(\omega)=\sum_{p=1}^{n-1} E_{p}^{(q)} y_{p} \quad(q=1, \ldots, n-1) .
$$

Moreover, since the matrix $\left(E_{p}^{(q)}\right)$ is regular, if we take $C_{1}, \ldots, C_{n-1}>0$ sufficiently small then $(43)$ holds for $\left(y_{1}, \ldots, y_{n-1}\right)$ which satisfy

$$
0 \leq W_{q}(\omega)<C_{q} \quad(q=1, \ldots, n-1) .
$$


In fact, all $\left(y_{1}, \ldots, y_{n-1}\right)$ which satisfy (43) form a region in $\mathbb{R}^{n-1}$ which contains 0 . Since this region is not empty, it contains a neighbourhood $U$ of 0 . On the other hand, since $\left(E_{p}^{(q)}\right)$ is regular, the set of all $\left(y_{1}, \ldots, y_{n-1}\right)$ which satisfy (44) is a bounded domain which touches 0 . By taking $C_{1}, \ldots, C_{n-1}$ $>0$ small enough, the region given by (44) can be contained in $U$. Therefore (43) follows from (44) for such $C_{q}$. Without loss of generality we may suppose that $C_{q}<1$. Let us fix such a $C_{q}$.

The formula in Proposition 6 is concerned with the number of prime ideal numbers. But we need a formula for prime numbers. To deduce it from Proposition 6, we prove two lemmas.

Lemma 5. If we take suitable $\left\{C_{q}^{(1)}\right\},\left\{C_{q}^{(2)}\right\}$ such that $0<C_{q}^{(1)}<C_{q}^{(2)}<$ $C_{q}$ then for any $\sigma \in G \backslash\{1\}$ there exists no $\omega \in K$ which satisfies

$$
C_{q}^{(1)} \leq W_{q}(\omega)<C_{q}^{(2)} \quad(q=1, \ldots, n-1)
$$

and

$$
C_{q}^{(1)} \leq W_{q}(\sigma(\omega))<C_{q}^{(2)} \quad(q=1, \ldots, n-1)
$$

at the same time.

Proof. We consider the region given by

$$
0 \leq \sum_{p=1}^{n} E_{p}^{(q)} x_{p}<C_{q} \quad(q=1, \ldots, n-1) .
$$

Let $\left(x_{1}^{(0)}, \ldots, x_{n}^{(0)}\right)$ be an inner point in this region such that $x_{i}^{(0)} \neq x_{j}^{(0)}$ $(i \neq j)$. Let $m=\max _{i \neq j}\left|x_{i}^{(0)}-x_{j}^{(0)}\right|$. We fix $\delta>0$ such that $\delta<m / 4$ and that the neighbourhood

$$
U_{\delta}=\left\{\left(x_{1}, \ldots, x_{n}\right)|| x_{q}^{(0)}-x_{q} \mid<\delta\right\}
$$

is contained in the region defined by (45). For $\sigma \in G$, we set

$$
U_{\delta}^{\sigma}=\left\{\left(x_{\sigma(1)}, \ldots, x_{\sigma(n)}\right) \mid\left(x_{1}, \ldots, x_{n}\right) \in U_{\delta}\right\} .
$$

By the definition of $\delta$, we have $U_{\delta} \cap U_{\delta}^{\sigma}=\emptyset$ for any $\sigma \neq 1$. Moreover, if we set

$$
U_{\delta}(t)=\left\{\left(x_{1}-t, \ldots, x_{n}-t\right) \mid\left(x_{1}, \ldots, x_{n}\right) \in U_{\delta}\right\}
$$

for $t \in \mathbb{R}$, then for any $\sigma \in G, \sigma \neq 1$, we have

$$
U_{\delta}^{\sigma} \cap U_{\delta}(t)=\emptyset .
$$

In fact if $\left(x_{1}, \ldots, x_{n}\right) \in U_{\delta}^{\sigma} \cap U_{\delta}(t)$ then

$$
x_{q} \in I_{q, \delta}=\left[x_{q}^{(0)}-\delta, x_{q}^{(0)}+\delta\right] \text { and } x_{q}+t \in I_{\sigma(q), \delta} .
$$

By the definition of $\delta$, it is impossible that more than two elements of $\left\{x_{q}+t \mid\right.$ $1 \leq q \leq n\}$ belong to one $I_{\sigma(q), \delta}$. Hence there exists a $q_{0}\left(1 \leq q_{0} \leq n\right)$ such that $I_{q_{0}, \delta}$ contains no $x_{\sigma(q)}+t(1 \leq q \leq n)$. This is a contradiction. 
We set

$$
A_{\delta}=\left\{\left(x_{1}+t, \ldots, x_{n}+t\right) \mid t \in \mathbb{R},\left(x_{1}, \ldots, x_{n}\right) \in U_{\delta}\right\}=\bigcup_{t \in \mathbb{R}} U_{\delta}(t) .
$$

This is a tube generated by translating $U_{\delta}$ along the vector $\overrightarrow{(1, \ldots, 1)}$. By the above argument $A_{\delta} \cap A_{\delta}^{\sigma}=\emptyset$ for any $\sigma \in G$.

On the other hand consider the region given by

$$
C_{q}^{(1)} \leq \sum_{p=1}^{n} E_{p}^{(q)} x_{q}<C_{q}^{(2)} \quad(q=1, \ldots, n-1)
$$

This is also a tube parallel to $\overrightarrow{(1, \ldots, 1)}$. By taking suitable $0<C_{q}^{(1)}, C_{q}^{(2)} \leq$ $C_{q}$, this region is contained in $A_{\delta}$. Hence (46) gives the assertion.

LEMma 6. Let $\omega \in K$ be a prime ideal number which satisfies the condition

$$
(* 2) \quad\left\{\begin{array}{l}
\omega \equiv 1\left(\bmod \widetilde{\tilde{f}^{\prime}}\right), \\
C_{q}^{(1)} \leq W_{q}(\omega)<C_{q}^{(2)} \quad(q=1, \ldots, n-1)
\end{array}\right.
$$

and $\mathfrak{p}=(\omega)$. If $\omega^{\prime}$ is a prime ideal number which satisfies $(* 2)$ and $\left(\omega^{\prime}\right)=\mathfrak{p}$, then $\omega^{\prime}=\omega$.

Proof. Since $\omega \equiv 1\left(\bmod \widetilde{\mathfrak{f}^{\prime}}\right)$ and $\left(\omega^{\prime}\right)=\mathfrak{p}$, we have $\omega^{\prime}=\varepsilon \omega$ for some $\varepsilon \in U\left(\widetilde{\mathfrak{f}^{\prime}}\right)$. We put $\varepsilon=\eta_{1}^{a_{1}} \ldots \eta_{n-1}^{a_{n-1}}$. By the definition $(33)$ of $W_{q}(\omega)$ we have

$$
W_{q}(\varepsilon \omega)=W_{q}(\omega)+a_{1} W_{q}\left(\eta^{(1)}\right)+\ldots+a_{n-1} W_{q}\left(\eta^{(n-1)}\right)
$$

and

$$
W_{q}\left(\eta^{(p)}\right)= \begin{cases}1 & (p=q) \\ 0 & (p \neq q)\end{cases}
$$

Hence $a_{1}=\ldots=a_{n-1}=0$. Thus $\omega^{\prime}=\omega$.

Now we deduce Proposition 5 from Proposition 6 and the above two lemmas. In view of Proposition 6, the number of prime ideal numbers $\omega$ satisfying the condition

$$
(* 3) \quad\left\{\begin{array}{l}
|N(\omega)| \leq x, \quad \omega \equiv 1\left(\bmod \tilde{\mathfrak{f}}^{\prime}\right) \\
C_{q}^{(1)} \leq W_{q}(\omega)<C_{q}^{(2)} \quad(q=1, \ldots, n-1)
\end{array}\right.
$$

is

$$
\prod_{q=1}^{n-1}\left(C_{q}^{(2)}-C_{q}^{(1)}\right) \frac{1}{h\left(\mathfrak{f}^{\prime}\right)} \int_{2}^{x} \frac{d t}{\log t}+O\left(x e^{-c \sqrt{\log x}}\right) .
$$

By Lemma 6 there is a one-to-one correspondence between prime ideal num- 
bers which satisfy $(* 3)$ and prime ideals $\mathfrak{p}$ which satisfy the condition

$$
(* 4) \quad\left\{\begin{array}{l}
N(\mathfrak{p}) \leq x, \quad \mathfrak{p} \in P_{\mathfrak{f}^{\prime}}, \\
\text { there exists an } \omega \in K \text { such that }(\omega)=\mathfrak{p}, \omega \equiv 1\left(\bmod \widetilde{\mathfrak{f}}^{\prime}\right) \\
\text { and } C_{q}^{(1)} \leq W_{q}(\omega)<C_{q}^{(2)}(q=1, \ldots, n-1) .
\end{array}\right.
$$

Hence (47) also gives the number of prime ideals which satisfy $(* 4)$. The number of prime ideals whose norm is less than $x$ and degree is greater than two is

$$
\ll \sum_{p^{2} \leq x} 1+\ldots+\sum_{p^{n} \leq x} 1 \ll \pi(\sqrt{x}) \ll O\left(x e^{-c \sqrt{\log x}}\right) .
$$

Hence we may add to $(* 4)$ the condition that the degree is 1 . We denote the resulting condition by $(* 5)$. Taking into account Lemma 5 , if the prime number $p$ completely splits in $K$ as in $(* 1)$ and $\mathfrak{p}_{1}$ satisfies $(* 5)$ then none of $\mathfrak{p}_{j}$ satisfies $(* 5)$. Thus there exists a one-to-one correspondence between prime ideals which satisfy $(* 5)$ and prime numbers which satisfy the condition

$$
(* 6) \quad\left\{\begin{array}{l}
p \leq x, \quad p=\mathfrak{p}_{1} \ldots \mathfrak{p}_{n}, \mathfrak{p}_{i} \in P_{\tilde{\mathfrak{f}}^{\prime}}, \\
\text { there exists only one } \mathfrak{p}_{i} \text { which satisfies }(* 5) .
\end{array}\right.
$$

Hence the number of prime numbers satisfying $(* 6)$ is given by $(47)$. If $p$ satisfies $(* 6)$, then by (42) and (44) we have $\left|\alpha_{p}\right| \geq n-\varepsilon$. This completes the proof for $K$ totally real.

Next we consider the case of $K$ totally imaginary Galois. Since $n=2 r_{2}$ and $r_{1}=0$, for $\mathfrak{a}=(\alpha)$ with $\alpha \equiv 1(\bmod \widetilde{\mathfrak{f}})$,

$$
\chi(\mathfrak{a})=\chi_{\infty}(\alpha)=\prod_{q=1}^{r_{2}}\left|\alpha^{(q)}\right|^{i v_{q}} \prod_{p=1}^{r_{2}}\left(\frac{\alpha^{(p)}}{\left|\alpha^{(p)}\right|}\right)^{a_{p}} .
$$

Let $\mathfrak{f}^{\prime}=(N(\mathfrak{f}))$. If a prime number $p$ satisfies the condition

$$
(* * 1) \quad\left\{\begin{array}{l}
p \text { completely splits in } K \text { as } p=\mathfrak{p}_{1} \ldots \mathfrak{p}_{n}, \\
\mathfrak{p}_{i} \in P_{\mathfrak{f}^{\prime}},
\end{array}\right.
$$

then

$$
\alpha_{p}=\sum_{\sigma \in G} \chi(\sigma(\omega))
$$

where $\omega$ is a prime ideal number such that $(\omega)=\mathfrak{p}_{1}, \omega \equiv 1\left(\bmod \widetilde{\mathfrak{f}^{\prime}}\right)$ and $G=\operatorname{Gal}(K / \mathbb{Q})$.

We note that the set $\{\sigma(\omega) \mid \sigma \in G\}$ consists of $r_{2}$ pairs of complex conjugates. We denote them by

$$
\sigma(\omega), \quad \overline{\sigma(\omega)} \quad\left(\sigma \in G^{\prime}\right)
$$

where $G^{\prime}=G /\langle\tau\rangle$ and $\tau$ denotes the complex conjugate isomorphism. The prime ideal number $\omega$ corresponds to $\left(\omega^{(1)}, \ldots, \omega^{\left(r_{2}\right)}, \overline{\omega^{(1)}}, \ldots, \overline{\omega^{\left(r_{2}\right)}}\right)$. For 
$\sigma \in G^{\prime}, \sigma(\omega)$ corresponds to $\left(\omega^{\sigma(1)}, \ldots, \omega^{\sigma\left(r_{2}\right)}, \overline{\omega^{\sigma(1)}}, \ldots, \overline{\omega^{\sigma\left(r_{2}\right)}}\right)$ and $\sigma(\bar{\omega})$ corresponds to $\left.\overline{\left(\omega^{\sigma(1)}\right.}, \ldots, \overline{\omega^{\sigma\left(r_{2}\right)}}, \omega^{\sigma(1)}, \ldots, \omega^{\sigma\left(r_{2}\right)}\right)$. Hence by (48), for $\sigma \in G^{\prime}$ we have

$$
\left\{\begin{array}{l}
\chi_{\infty}(\sigma(\omega))=\prod_{q=1}^{r_{2}}\left|\omega^{(q)}\right|^{i v_{\sigma^{-1}(q)}} \prod_{p=1}^{r_{2}}\left(\frac{\alpha^{(p)}}{\left|\alpha^{(p)}\right|}\right)^{a_{\sigma^{-1}(p)}}, \\
\chi_{\infty}(\overline{\sigma(\omega)})=\prod_{q=1}^{r_{2}}\left|\omega^{(q)}\right|^{i v_{\sigma^{-1}(q)}} \prod_{p=1}^{r_{2}}\left(\frac{\alpha^{(p)}}{\left|\alpha^{(p)}\right|}\right)^{-a_{\sigma^{-1}(p)}} .
\end{array}\right.
$$

Hence, taking into account (49) and (50), for a prime number $p$ which satisfies $(* * 1)$ and a prime ideal number $\omega$ which satisfies the above condition for $p$ we have

$$
\begin{aligned}
\alpha_{p}= & \sum_{\sigma \in G^{\prime}}\left(\chi_{\infty}(\sigma(\omega))+\chi_{\infty}(\overline{\sigma(\omega)})\right) \\
= & \sum_{\sigma \in G^{\prime}} \prod_{q=1}^{r_{2}}\left|\omega^{(q)}\right|^{i v_{\sigma(q)}}\left\{\exp \left(i \sum_{p=1}^{r_{2}} a_{\sigma(p)} \arg \omega^{(p)}\right)\right. \\
& \left.+\exp \left(-i \sum_{p=1}^{r_{2}} a_{\sigma(p)} \arg \omega^{(p)}\right)\right\} \\
= & \sum_{\sigma \in G^{\prime}} \prod_{q=1}^{r_{2}}\left|\omega^{(q)}\right|^{i v_{\sigma(q)}}\left\{2 \cos \left(\sum_{p=1}^{r_{2}} a_{\sigma(p)} \arg \omega^{(p)}\right)\right\} \\
= & \prod_{q=1}^{r_{2}}\left|\omega^{(q)}\right|^{i v_{q}}\left\{2 \cos \left(\sum_{p=1}^{r_{2}} a_{p} \arg \omega^{(p)}\right)\right. \\
& \left.\quad+\sum_{\sigma \neq 1} 2 \cos \left(\sum_{p=1}^{r_{2}} a_{\sigma(p)} \arg \omega^{(p)}\right) \exp \left(i \sum_{q=1}^{r_{2}}\left(v_{\sigma(q)}-v_{q}\right) x_{q}\right)\right\} .
\end{aligned}
$$

Hence for such $p$ we have

$$
\begin{aligned}
\left|\alpha_{p}\right|=\mid 2 & \cos \left(\sum_{p=1}^{r_{2}} a_{p} \arg \omega^{(p)}\right) \\
& +\sum_{\sigma \neq 1} 2 \cos \left(\sum_{p=1}^{r_{2}} a_{\sigma(p)} \arg \omega^{(p)}\right) \exp \left(i \sum_{q=1}^{r_{2}}\left(v_{\sigma(q)}-v_{q}\right) x_{q}\right) \mid
\end{aligned}
$$

where we have set $x_{q}=\log \left|\omega^{(q)}\right|$.

Now we consider the conditions $\omega$ under which the right-hand side of (51) is sufficiently close to $n=2 r_{2}$.

By the definition of $\left(E_{p}^{(q)}\right)_{1 \leq p, q \leq r_{2}}$, we find that the matrix $\left(E_{p}^{(q)}\right)$ is regular and that $\sum_{p} E_{p}^{(q)}=0$ for any $q$. Hence, as in the totally real case, 
for any sufficiently small $\varepsilon>0$ there exist suitable $C_{q}>0\left(q=1, \ldots, r_{2}-1\right)$ such that if we suppose

$$
0 \leq W_{q}(\omega) \leq C_{q} \quad\left(q=1, \ldots, r_{2}-1\right)
$$

then

$$
\begin{gathered}
\left|\sum_{q=1}^{r_{2}}\left(v_{\sigma(q)}-v_{q}\right) x_{q}\right|<\varepsilon \quad\left(\sigma \in G^{\prime}, \sigma \neq 1\right), \\
\left|\exp \left\{i \sum_{q=1}^{r_{2}}\left(v_{\sigma(q)}-v_{q}\right) x_{q}\right\}-1\right|<\varepsilon \quad\left(\sigma \in G^{\prime}, \sigma \neq 1\right) .
\end{gathered}
$$

On the other hand, by (34),

$$
\Theta_{p}(\omega)=\frac{1}{2 \pi}\left\{\arg \omega^{(p)}-\sum_{j=1}^{r} \Theta_{j}^{(p)} W_{j}(\omega)\right\} \quad\left(p=1, \ldots, r_{2}\right) .
$$

For an $\omega$ which satisfies (52) we suppose that

$$
\left|\Theta_{p}(\omega)-\frac{1}{2 \pi} \arg \omega^{(p)}\right|<\varepsilon \quad\left(p=1, \ldots, r_{2}\right) .
$$

If

$$
0 \leq \Theta_{p}(\omega)<b_{p} \quad\left(p=1, \ldots, r_{2}\right)
$$

for sufficiently small $b_{1}, \ldots, b_{r_{2}}>0$, then by (55) we obtain $\left|\arg \omega^{(p)}\right|<\varepsilon$ $\left(p=1, \ldots, r_{2}\right)$ and

$$
\left|2 \cos \left(\sum_{p=1}^{r_{2}} a_{\sigma(p)} \arg \omega^{(p)}\right)-2\right|<\varepsilon \quad\left(\sigma \in G^{\prime}\right) .
$$

Therefore for any $\omega$ satisfying (52) and (56), in view of (51), (54) and (57), we have

$$
\left|\sum_{\sigma \in G} \chi_{\infty}(\sigma(\omega))-n\right|<\varepsilon .
$$

We may suppose that $C_{q}<1$ and $b_{p}<\min \left\{3 / 8,1 / w\left(\widetilde{\mathfrak{f}}^{\prime}\right)\right\}$.

Similarly to the previous case, we prove the following two lemmas:

Lemma 7. If we choose suitable $C_{q}^{(1)}, C_{q}^{(2)}$ which satisfy $0<C_{q}^{(1)}<$ $C_{q}^{(2)}<C_{q}$ then for any $\sigma \neq 1$ there is no $\omega \in K$ such that $\omega$ and $\sigma(\omega)$ satisfy the condition

$$
(* * 2) \quad\left\{\begin{array}{l}
C_{q}^{(1)} \leq W_{q}(\omega)<C_{q}^{(2)} \quad\left(q=1, \ldots, r_{2}-1\right), \\
b_{p} / 2 \leq \Theta_{p}(\omega)<b_{p} \quad\left(p=1, \ldots, r_{2}\right)
\end{array}\right.
$$

at the same time. 
Proof. Since $W_{q}(\omega)$ is stable under the complex conjugate $\tau$, it is enough to prove the lemma for $\sigma \in G^{\prime}$ and $\tau$. We take $0<C_{q}^{\prime} \leq C_{q}$ such that

$$
\max _{0 \leq W_{q}(\omega)<C_{q}^{\prime}}\left|\sum_{j=1}^{r_{2}-1} \Theta_{j}^{(p)} W_{j}(\omega)\right|<\frac{2 \pi}{3} b_{p} \quad\left(p=1, \ldots, r_{2}\right) .
$$

Similarly to the proof of Lemma 5 , if we choose suitable $0 \leq C_{q}^{(1)}<C_{q}^{(2)}<$ $C_{q}^{\prime}\left(q=1, \ldots, r_{2}-1\right)$ then for $\omega \in K$ satisfying

$$
C_{q}^{(1)} \leq W_{q}(\omega)<C_{q}^{(2)} \quad\left(q=1, \ldots, r_{2}-1\right),
$$

$\sigma(\omega)$ does not satisfy $(60)$ for any $\sigma \neq 1, \tau$.

Next we consider $\tau$. Since $W_{q}(\bar{\omega})=W_{q}(\omega)$, the first condition in $(* * 2)$ is also valid for $W_{q}(\bar{\omega})$. We show that the second condition does not hold for $W_{q}(\bar{\omega})$. Under $(60)$ we suppose that

$$
b_{p} / 2 \leq \Theta_{p}(\omega)<b_{p} \quad\left(p=1, \ldots, r_{2}\right) .
$$

Then by (34) and (59) we obtain

$$
\frac{b_{p}}{6} \leq \frac{1}{2 \pi} \arg \omega^{(p)}<\frac{4}{3} b_{p} \quad\left(p=1, \ldots, r_{2}\right) .
$$

This and (58) give

$$
-\frac{5}{3} b_{p} \leq \Theta_{p}(\omega) \leq \frac{b_{p}}{6} \quad\left(p=1, \ldots, r_{2}\right) .
$$

Since $b_{p}<3 / 8$, the intervals $\left[b_{p} / 2, b_{p}\right]$ and $\left[-5 b_{p} / 3, b_{p} / 6\right]$ do not intersect. Therefore if $\omega$ satisfies (60) and (61) then $\bar{\omega}$ does not satisfy (61).

Lemma 8. Let $\omega \in K$ be a prime ideal number which satisfies the condition

$$
(* * 3)\left\{\begin{array}{l}
\omega \equiv 1\left(\bmod \tilde{\mathfrak{f}}^{\prime}\right), \\
C_{q}^{(1)} \leq W_{q}(\omega)<C_{q}^{(2)} \quad\left(q=1, \ldots, r_{2}-1\right), \\
b_{p} / 2 \leq \Theta_{p}(\omega)<b_{p} \quad\left(p=1, \ldots, r_{2}\right)
\end{array}\right.
$$

and $\mathfrak{p}=(\omega)$. If $\omega^{\prime}$ is a prime ideal number which satisfies $(* * 3)$ and $\left(\omega^{\prime}\right)=\mathfrak{p}$, then $\omega^{\prime}=\omega$.

Proof. Similarly to the proof of Lemma 6, we put

$$
\omega^{\prime}=\varepsilon \omega, \quad \varepsilon=\varrho^{a} \eta_{1}^{b_{1}} \ldots \eta_{r_{2}-1}^{b_{r_{2}-1}} \quad\left(a, b_{i} \in \mathbb{Z}\right)
$$

where $\varrho=\exp \left(2 \pi / w\left(\widetilde{\mathfrak{f}^{\prime}}\right)\right)$. By the second condition above we have

$$
b_{1}=\ldots=b_{r_{2}-1}=0 \text {. }
$$

By the definition of $\Theta_{p}(\omega)$, we have

$$
\Theta_{p}\left(\varrho^{a} \omega\right)=a \Theta_{p}(\varrho)+\Theta_{p}(\omega)
$$


and

$$
\Theta_{p}(\varrho)=\frac{1}{2 \pi} \arg \varrho^{(p)}-\frac{1}{2 \pi} \sum_{j=1}^{r_{2}-1} \Theta_{j}^{(p)} W_{j}(\varrho)=\frac{1}{2 \pi} \arg \varrho^{(p)}=\frac{k_{p}}{\left.w \widetilde{\mathfrak{f}^{\prime}}\right)},
$$

where $k_{p} \in \mathbb{Z}-\{0\}$, hence

$$
\left|\Theta_{p}(\omega)\right| \geq 1 / w\left(\widetilde{\tilde{f}^{\prime}}\right)>b_{p} .
$$

Thus by the third condition we obtain $a=0$.

Now we deduce Proposition 5 from these two lemmas and Proposition 6. In view of Proposition 6, the number of prime ideal numbers which satisfy the condition

$$
(* * 4) \quad\left\{\begin{array}{l}
|N(\omega)| \leq x, \quad \omega \equiv 1\left(\bmod \widetilde{\mathfrak{f}}^{\prime}\right) \\
C_{q}^{(1)} \leq W_{q}(\omega)<C_{q}^{(2)} \quad\left(q=1, \ldots, r_{2}-1\right), \\
b_{p} / 2 \leq \Theta_{p}(\omega)<b_{p} \quad\left(p=1, \ldots, r_{2}\right)
\end{array}\right.
$$

is given by

$$
\prod_{q=1}^{r_{2}-1}\left(C_{q}^{(2)}-C_{q}^{(1)}\right) \prod_{p=1}^{r_{2}} b_{p} \frac{w\left(\widetilde{\mathfrak{f}^{\prime}}\right)}{2^{r_{2}} h\left(\widetilde{\mathfrak{f}^{\prime}}\right)} \int_{2}^{x} \frac{d t}{\log t}+O\left(x e^{-c \sqrt{\log x}}\right) .
$$

By Lemma 8, there is a one-to-one correspondence between prime ideal numbers $\omega$ satisfying $(* * 4)$ and prime ideals $\mathfrak{p}$ which satisfy the condition

$$
(* * 5) \quad\left\{\begin{array}{l}
N(\mathfrak{p}) \leq x, \quad \mathfrak{p} \in P_{\tilde{\mathfrak{f}}^{\prime}}, \\
\text { there exists an } \omega \in K \text { for } \mathfrak{p} \text { such that }(\omega)=\mathfrak{p}, \omega \equiv 1\left(\bmod \widetilde{\mathfrak{f}}^{\prime}\right), \\
C_{q}^{(1)} \leq W_{q}(\omega)<C_{q}^{(2)} \quad\left(q=1, \ldots, r_{2}-1\right) \\
b_{p} / 2 \leq \Theta_{p}(\omega)<b_{p} \quad\left(p=1, \ldots, r_{2}\right)
\end{array}\right.
$$

Hence the formula (62) also gives the number of prime ideals satisfying $(* * 5)$. Similarly to the previous case, we may add to $(* * 5)$ the condition that the degree of $\mathfrak{p}$ is 1 .

Taking into account Lemma 7 , if a prime $p$ completely splits in $K$ and $\mathfrak{p}_{1}$ satisfies the condition $(* * 5)$ then none of the other $\mathfrak{p}_{i}$ satisfies $(* * 5)$. Thus there is one-to-one correspondence between prime ideals which satisfy $(* * 5)$ and prime numbers which satisfy the condition

$$
(* * 6) \quad\left\{\begin{array}{l}
p \leq x \text { completely splits as } p=\mathfrak{p}_{1} \ldots \mathfrak{p}_{n}, \\
\text { only one } \mathfrak{p}_{i} \text { satisfies }(* * 5) .
\end{array}\right.
$$

Hence the number of prime numbers satisfying $(* * 6)$ is given by (62). In view of (52), (56) and (58), for $p$ satisfying (**6) we have $\left|\alpha_{p}\right| \geq n-\varepsilon$. Thus we have completed the proof of Proposition 5 for $K$ Galois.

Now we consider the general case. The proof follows Professor Okazaki's idea. Let $L / \mathbb{Q}$ be the Galois closure of $K,[L: K]=N, \operatorname{Gal}(L / \mathbb{Q})=H$, and 
$\operatorname{Gal}(L / K)=I$; let $R_{1}$ be the number of real embeddings of $L$ into $\mathbb{C}, 2 R_{2}$ be the number of imaginary embeddings and $R=R_{1}+R_{2}-1$. We denote these embeddings by

$$
L \ni \Omega \mapsto \Omega^{(q)} \in L^{(q)}=L \quad(q=1, \ldots, n N) .
$$

For an ideal $\mathfrak{f}$ in $K$ we set $\mathfrak{f}_{L}=\left(N_{K / \mathbb{Q}}(\mathfrak{f})\right)_{L}$, where ()$_{L}$ means a principal ideal in $L$. Suppose that a prime number $p$ completely splits in $L$ as follows:

$$
\left\{\begin{array}{l}
p=\mathfrak{P}_{1} \ldots \mathfrak{P}_{n N}, \\
\mathfrak{P}_{1}=(\Omega)_{L}, \quad \Omega \in L, \quad \Omega \equiv 1\left(\bmod \tilde{\mathfrak{f}}_{L}\right) .
\end{array}\right.
$$

According to the above two cases, for any $\mathfrak{P}_{i}$ there exists $\sigma \in H$ such that

$$
\mathfrak{P}_{i}=(\sigma(\Omega))_{L}, \quad \sigma(\Omega) \equiv 1\left(\bmod \tilde{\mathfrak{f}}_{L}\right) .
$$

Let $\mathfrak{p}_{1}$ be the norm of $\mathfrak{P}_{1}$ over $L / K$, i.e.

$$
\mathfrak{p}_{1}=\prod_{\sigma \in I} \mathfrak{P}_{1}^{\sigma}=\left(\omega_{1}\right), \quad \omega_{1}=N_{L / K}(\Omega) \in K .
$$

Then $\mathfrak{p}_{1}$ is a prime ideal in $K$ and $\omega_{1} \equiv 1\left(\bmod \widetilde{\mathfrak{f}}_{L}^{\prime}\right)$ by $(62)$. Let $\mathfrak{P}_{2}$ be one of the $\mathfrak{P}_{i}$ 's which do not divide $\mathfrak{p}_{1}$. We define $\mathfrak{p}_{2}=\left(\omega_{2}\right)=N_{L / K}\left(\mathfrak{P}_{2}\right)$. Then $\mathfrak{p}_{2}$ is also a prime ideal in $K$ and satisfies $\omega_{2} \equiv 1\left(\bmod \widetilde{\mathfrak{f}}_{L}^{\prime}\right)$. Therefore a prime $p$ satisfying (!1) splits completely in $K$ as follows:

$$
\left\{\begin{array}{l}
p=\mathfrak{p}_{1} \ldots \mathfrak{p}_{n}, \\
\mathfrak{p}_{i}=\left(\omega_{i}\right) \in P_{\widetilde{\mathfrak{f}}^{\prime}}, \quad \omega_{i} \equiv 1\left(\bmod \widetilde{\mathfrak{f}}^{\prime}\right) .
\end{array}\right.
$$

By the definitions (2) and (4), for a prime $p$ satisfying (!1) we have

$$
\begin{aligned}
\alpha_{p} & =\sum_{i=1}^{n} \chi\left(\mathfrak{p}_{i}\right)=\sum_{i=1}^{n} \chi_{\infty}\left(\omega_{i}\right) \\
& =\sum_{i=1}^{n} \prod_{q=1}^{r_{1}+r_{2}}\left|\omega_{i}^{(q)}\right|^{i v_{q}} \prod_{p=r_{1}+1}^{r_{1}+r_{2}}\left(\frac{\omega_{i}^{(p)}}{\left|\omega_{i}^{(p)}\right|}\right)^{a_{p}} \\
& =\sum_{i=1}^{n} \prod_{q=1}^{r}\left(\frac{\left|\omega_{i}^{(q)}\right|}{\left|\omega_{i}^{\left(r_{1}+r_{2}\right)}\right|}\right)^{i v_{q}} \prod_{p=r_{1}+1}^{r_{1}+r_{2}}\left(\frac{\omega_{i}^{(p)}}{\left|\omega_{i}^{(p)}\right|}\right)^{a_{p}} .
\end{aligned}
$$

Since $\left|N_{K}\left(\omega_{i}\right)\right|=\prod_{1 \leq q \leq n}\left|\omega_{i}^{(q)}\right|=p$, if we take $\varepsilon>0$ sufficiently small, then $\left|\alpha_{p}\right|$ is sufficiently close to $n$ under the condition

$$
\left\{\begin{array}{l}
1-\varepsilon<\left|\omega_{i}^{(q)}\right| / \sqrt[n]{p}<1+\varepsilon \\
-\varepsilon<\arg \omega_{i}^{(p)}<\varepsilon
\end{array}\right.
$$


We set $\varepsilon^{\prime}=1-(1-\varepsilon)^{1 / N}, \varepsilon^{\prime \prime}=(1+\varepsilon)^{1 / N}-1$ and suppose that

$$
\left\{\begin{array}{l}
p=\mathfrak{P}_{1} \ldots \mathfrak{P}_{n N}, \\
\mathfrak{P}_{1}=(\Omega)_{L}, \quad \Omega \equiv 1\left(\bmod \widetilde{\mathfrak{f}}_{L}\right), \\
1-\varepsilon^{\prime}<\left|\Omega^{(q)}\right| / \sqrt[n N]{p}<1+\varepsilon^{\prime \prime} \quad\left(q=1, \ldots, R_{1}+R_{2}\right) \\
-\varepsilon / N<\arg \Omega^{(p)}<\varepsilon / N \quad\left(p=R_{1}+1, \ldots, R_{1}+R_{2}\right) .
\end{array}\right.
$$

We note that any $\omega_{i}^{(q)}$ is a product of $N$ conjugates of $\Omega$. Hence

$$
\left(1-\varepsilon^{\prime}\right)^{N}<\omega_{i}=\prod \frac{\left|\Omega^{(q)}\right|}{\sqrt[n N]{p}}<\left(1+\varepsilon^{\prime \prime}\right)^{N},
$$

thus

$$
1-\varepsilon<\frac{\left|\omega_{i}^{(q)}\right|}{\sqrt[n]{p}}<1+\varepsilon
$$

and

$$
-\varepsilon<\arg \omega_{i}^{(p)}=\sum \arg \Omega^{(q)}<\varepsilon .
$$

Therefore primes $p$ satisfying (!4) also satisfy (!1) and (!2).

Let $\eta_{1}, \ldots, \eta_{R} \in L$ be fundamental units of the unit group in $L$ modulo $\widetilde{\mathfrak{f}}_{L}$. We define $W_{q}, \Theta_{p}$ in (33) and (34) for them. If we set

$$
Y_{q}=\log \left|\Omega^{(q)}\right|-\log \left|\Omega^{\left(R_{1}+R_{2}\right)}\right| \quad(q=1, \ldots, R)
$$

then the third relation in (!4) means

$$
-\varepsilon<Y^{(q)}<\varepsilon .
$$

Hence similarly to the second case, if we choose suitable $C_{q}>0(q=$ $1, \ldots, R)$ and $B_{p}>0\left(p=R_{1}+1, \ldots, R_{1}+R_{2}\right)$ then the third and fourth relations in (!4) hold for prime ideal numbers $\Omega$ which satisfy

$$
\begin{cases}0<W_{q}(\Omega)<C_{q} & (q=1, \ldots, R), \\ 0<\Theta_{p}(\Omega)<B_{p} & \left(p=R_{1}+1, \ldots, R_{1}+R_{2}\right) .\end{cases}
$$

By Proposition 6, Lemma 7 and Lemma 8, we can calculate the number of primes $p$ satisfying

$$
(! 5) \quad\left\{\begin{array}{l}
p \leq x, \quad p=\mathfrak{P}_{1} \ldots \mathfrak{P}_{n N}, \\
\text { only one } \mathfrak{P}_{i} \text { satisfies } \mathfrak{P}_{i}=(\Omega), \\
\Omega \equiv 1\left(\bmod \widetilde{\mathfrak{f}}_{L}\right), \\
C_{q}^{(1)}<W_{q}(\Omega)<C_{q}^{(2)} \quad(q=1, \ldots, R), \\
B_{p}^{(1)}<\Theta_{p}(\Omega)<B_{p}^{(2)} \quad\left(p=R_{1}+1, \ldots, R_{1}+R_{2}\right),
\end{array}\right.
$$

where $0<C_{q}^{(1)}<C_{q}^{(2)}<C_{q}, 0<B_{p}^{(1)}<B_{p}^{(2)}<B_{p}$. By (!1) and (!2), $\left|\alpha_{p}\right| \geq n-\varepsilon$ for such $p$.

This completes the proof of Proposition 5, and therefore, the proof of the Theorem. 


\section{References}

[1] B. Bagchi, The statistical behaviour and universality properties of the Riemann zetafunction and other allied Dirichlet series, Ph.D. thesis, Indian Statistical Institute, Calcutta, 1981.

[2] E. Hecke, Über eine neue Art von Zetafunktionen, Math. Z. 6 (1920), 11-51.

[3] A. A. Karatsuba and S. M. Voronin, The Riemann Zeta-Function, de Gruyter, 1992.

[4] A. Laurinčikas, Limit Theorems for the Riemann Zeta-Function, Kluwer, Dordrecht, 1996.

[5] -, On the Matsumoto zeta-function, Acta Arith. 84 (1998), 1-16.

[6] -, On limit distribution of the Matsumoto zeta-function, Lithuanian Math. J. 36 (1996), 371-387.

[7] H. Mishou, The universality theorem for $L$-functions associated with ideal class characters, Acta Arith. 98 (2001), 395-410.

[8] T. Mitsui, Kaisekitekiseisuuron, Iwanami, 1989 (in Japanese).

[9] —, Generalized prime number theorem, Japan. J. Math. 26 (1956), 1-42.

[10] H. S. A. Potter, The mean values of certain Dirichlet series 1, Proc. London Math. Soc. 46 (1940), 467-478.

[11] A. Reich, Werteverteilung von Zetafunktionen, Arch. Math. (Basel) 34 (1980), 440451.

[12] S. M. Voronin, A theorem on the "universality" of the Riemann zeta-function, Izv. Akad. Nauk SSSR Ser. Mat. 39 (1975), 475-486 (in Russian); Math. USSR-Izv. 9 (1975), 443-453.

[13] J. L. Walsh, Interpolation and Approximation by Rational Functions in the Complex Domain, Amer. Math. Soc. Colloq. Publ. 20, Amer. Math. Soc., Providence, RI, 1960.

Graduate School of Mathematics

Nagoya University

Chikusa-ku, Nagoya 464-8602, Japan

E-mail: m98018a@math.nagoya-u.ac.jp

Received on 19.7.2002

and in revised form on 11.10.2002 